\title{
The factor Xa inhibitor rivaroxaban reduces cardiac dysfunction in a mouse model of myocardial infarction
}

\author{
Michael F. Bode ${ }^{\mathrm{a}}$, Alyson C. Auriemma ${ }^{\mathrm{b}}$, Steven P. Grover ${ }^{\mathrm{b}}$, Yohei Hisada ${ }^{\mathrm{b}}$, Alex Rennie ${ }^{\mathrm{b}}$, \\ Weeranun D. Bode ${ }^{\mathrm{a}}$, Rashi Vora ${ }^{\mathrm{d}}$, Saravanan Subramaniam ${ }^{\mathrm{b}, \mathrm{c}}$, Brian Cooley ${ }^{\mathrm{c}}$, \\ Patricia Andrade-Gordon ${ }^{\mathrm{e}}$, Silvio Antoniak ${ }^{\mathrm{c}}$, Nigel Mackman ${ }^{\mathrm{b}, \mathrm{c}, *}$ \\ a Division of Cardiology, Department of Medicine, McAllister Heart Institute, University of North Carolina at Chapel Hill, Chapel Hill, NC, USA \\ b Division of Hematology and Oncology, McAllister Heart Institute, Department of Medicine, University of North Carolina at Chapel Hill, Chapel Hill, NC, USA \\ ${ }^{\mathrm{c}}$ Department of Pathology and Laboratory Medicine, University of North Carolina at Chapel Hill, Chapel Hill, NC, USA \\ ${ }^{\mathrm{d}}$ Department of Medicine, University of North Carolina at Chapel Hill, Chapel Hill, NC, USA \\ e Janssen Research and Development, LLC, Spring House, PA, USA
}

\section{A R T I C L E I N F O}

Keywords:

Cardiac remodeling

Coagulation

Factor Xa

Myocardial infarction

Protease-activated receptor

Rivaroxaban

\begin{abstract}
A B S T R A C T
Introduction: Rivaroxaban selectively inhibits factor Xa (FXa), which plays a central role in blood coagulation. In addition, FXa activates protease-activated receptor-2 (PAR-2). We have shown that PAR-2 ${ }^{-/-}$mice exhibit less cardiac dysfunction after cardiac injury.

Material and methods: Wild-type (WT) and PAR-2 $2^{-/-}$mice were subjected to left anterior descending artery (LAD) ligation to induce cardiac injury and heart failure. Mice received either placebo or rivaroxaban chow either starting at the time of surgery or 3 days after surgery and continued up to 28 days. Cardiac function was measured by echocardiography pre-surgery and 3, 7 and 28 days after LAD ligation. We also measured anticoagulation, intravascular thrombi, infarct size, cardiac hypertrophy and inflammation at various times.

Results: Rivaroxaban increased the prothrombin time and inhibited the formation of intravascular thrombi in mice subjected to LAD ligation. WT mice receiving rivaroxaban immediately after surgery had similar infarct sizes at day 1 as controls but exhibited significantly less impairment of cardiac function at day 3 and beyond compared to the placebo group. Rivaroxaban also inhibited the expansion of the infarct at day 28. Rivaroxaban did not significantly affect the expression of inflammatory mediators or a neutrophil marker at day 2 after LAD ligation. Delaying the start of rivaroxaban administration until 3 days after surgery failed to preserve cardiac function. In addition, rivaroxaban did not reduce cardiac dysfunction in PAR-2 $2^{-1-}$ mice.

Conclusions: Early administration of rivaroxaban preserves cardiac function in mice after LAD ligation.
\end{abstract}

\section{Introduction}

Myocardial infarction (MI) leads to an abrupt loss of cardiomyocytes due to necrosis and apoptosis. The border zone around the infarct contains cardiomyocytes that may ultimately die leading to an expansion of the initial infarct. For instance, formation of intravascular thrombi may reduce blood flow in the border zone. The change in loading conditions after a MI triggers a compensatory cardiac remodeling in the myocardium [1]. Early remodeling begins within hours after the initial infarct [1]. The ensuing cardiac remodeling of the infarcted area results in ventricular dilation, thinning of the affected wall and an increase in wall stress that also induces remodeling of the noninfarcted remote myocardium to preserve stroke volume [2,3].
However, myocytes that have undergone pathologic hypertrophy to counteract unfavorable loading conditions eventually die leading to heart failure [1].

There are two animal models of MI in which the left anterior descending (LAD) coronary artery is either permanently ligated (LAD model) or is transiently ligated (ischemia-reperfusion [I/R] model). Tissue factor (TF) and coagulation proteases have been shown to contribute to cardiac injury. For instance, inhibition of the TF-factor (F) VIIa complex, factor Xa (FXa) or thrombin has been shown to reduce infarct size and no-reflow in rabbit, rat and mouse I/R models [4-8]. Inhibitors of TF or thrombin inhibitors also reduced inflammation, whereas the FXa inhibitor fondaparinux did not affect inflammation [4-7,9]. Immobilized fibrinogen can activate leukocytes by binding to

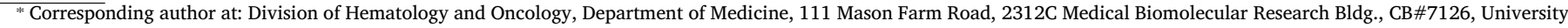
of North Carolina at Chapel Hill, Chapel Hill, NC 27599, USA.

E-mail address: nmackman@med.unc.edu (N. Mackman).
} 
the integrin $\alpha \mathrm{M} \beta 2$ [10]. In addition, a fibrin degradation product called E1 enhances I/R injury by facilitating the recruitment of neutrophils into the myocardium [11]. These studies indicate that the coagulation cascade contributes to cardiac injury after a MI.

Coagulation proteases and other proteases activate protease-activated receptors (PARs), which comprise of 4 members PAR-1 to 4 [12]. FXa activates both PAR-1 and PAR-2 whereas thrombin activates PAR1, PAR-3 and PAR-4 [12]. PAR-1, PAR-2 and PAR-4 have been shown to play a role in cardiac injury and remodeling in the cardiac I/R injury model $[6,13,14]$. We have shown that PAR-2 $2^{-/-}$mice have reduced inflammation and infarct size in an I/R injury model compared with controls [13]. We also found that PAR-2 $2^{-/-}$mice had less cardiac injury in the LAD model [15]. In contrast, the absence of PAR-1 did not affect the initial infarct size but reduced cardiac remodeling in an I/R injury model [6].

Neutrophils enhance cardiac injury after MI by releasing matrix metalloproteinases that contribute to infarct expansion by degrading intercellular collagen struts [16-18]. In addition, there is an increase in various inflammatory mediators, such as IL- $1 \beta$, IL- 6 , and TNF- $\alpha$, that contribute to cardiac injury $[19,20]$.

Rivaroxaban selectively inhibits FXa [21]. It is a FDA-approved oral anticoagulant used for the prevention and treatment of venous thromboembolism, and the prevention of stoke in patients with atrial fibrillation [22]. Rivaroxaban has been studied in a variety of animal models. It reduces thrombosis [23,24], and was associated with less bleeding compared to warfarin in a tissue-type plasminogen activator stroke model $[25,26]$. In addition, rivaroxaban reduces pressure-induced atrial fibrosis, atherosclerosis, diabetic nephropathy, neovascularization in diabetic mice and inflammation in sickle cell disease [27-32]. Interestingly, rivaroxaban reduced PAR-1 and PAR-2 expression in a stroke model and in diabetic nephropathy $[25,30]$. PAR-2 ${ }^{-/-}$ mice had reduced diabetic nephropathy and inflammation in the sickle cell disease model [30,32], which is similar to the phenotype of wildtype (WT) mice treated with rivaroxaban. These studies suggest that rivaroxaban may reduce PAR-2 signaling.

In this study, we examined the effects of rivaroxaban on cardiac function in mice after cardiac injury induced by LAD ligation. We chose the LAD model for these studies because it does not contain a reperfusion phase that affects the size of the initial infarct.

\section{Material and methods}

\subsection{Mice}

All studies were performed in accordance with the guidelines of the Institutional Animal Care and Use Committee of the University of North Carolina at Chapel Hill and in compliance with National Institutes of Health guidelines. We used male C57BL/6J (WT) mice as well as PAR$2^{-/-}$mice [33] and PAR-2 $2^{+/+}$(a sister line to the knock-out mice) between 8 and 12 weeks of age. Mice were fed rivaroxaban chow $(0.5 \mathrm{~g} / \mathrm{kg}$ chow) (Bayer AG, Leverkusen, Germany), which gives an estimated dose of $80 \mathrm{mg} / \mathrm{kg} /$ day based on normal feeding, or matching placebo chow (Dyets, Bethlehem, PA). Mice were given rivaroxaban chow immediately after recovery from anesthesia after LAD ligation or sham surgery. In a second experiment, rivaroxaban chow was started on day 3 after LAD ligation.

\subsection{LAD ligation}

LAD ligation was performed as previously described [15,34]. Briefly, mice were anesthetized with an intraperitoneal injection of ketamine and xylazine (100 and $15 \mathrm{mg} / \mathrm{kg}$, respectively). Mice were orally intubated to provide artificial ventilation $(140-180 \mu \mathrm{L}$ tidal volume, $130-140$ breaths/min). Following a left thoracotomy at the fourth intercostal space, the LAD was ligated with an 8-0 nylon surgical suture $2 \mathrm{~mm}$ from the distal tip of the left atrium. Following the occlusion, the chest was sutured and closed in 2 layers. Mice recovered on a water-jacketed warming blanket. Mice received buprenorphine ( $50 \mu \mathrm{g} / \mathrm{kg}$ subcutaneously) for post-operative analgesia. The surgeon was blinded to the genotype of the mice.

\subsection{Measurement of the plasma level of rivaroxaban}

Plasma rivaroxaban was measured by using a functional assay as described [35] with some modifications. Briefly, $100 \mu \mathrm{L}$ of chromogenic substrate (Liquid anti-Xa substrate, Diagnostica Stago, Parsippany, NJ) was added to $50 \mu \mathrm{L}$ mouse plasma samples (1:2 dilution) in imidazole buffer and incubated for $60 \mathrm{~s}$ before adding $100 \mu \mathrm{L}$ of STA ${ }^{\circledR}$ Liquid AntiXa (Diagnostica Stago). The change in optical density was determined at $405 \mathrm{~nm}$ from 90 to $120 \mathrm{~s}$. The logarithmic measurement was expressed as a function of the linear concentration. A standard curve was generated using a range of rivaroxaban concentrations $(5-640 \mathrm{ng} / \mathrm{mL})$ (Sigma-Aldrich, St Louis, MO). Imidazole buffer was used to dilute samples and standards. Results were expressed as a rivaroxaban concentration $(\mathrm{ng} / \mathrm{mL})$.

\subsection{Measurement of the prothrombin time}

Blood was collected into syringes containing sodium citrate (final concentration: $0.38 \%$, RICCA chemical company, Arlington, TX, USA) from the infrarenal vena cava. Plasma was prepared by centrifugation of the blood at $4500 \times g$ for $15 \mathrm{~min}$ and stored at $-80^{\circ} \mathrm{C}$. The prothrombin time (PT) was determined using a 1-stage clotting assay with mouse plasma, Thromboplastin D reagent (Thermo Fisher Scientific, Waltham, MA) and a Start 4 Clotting Machine [36] (Diagnostica Stago).

\subsection{Fibrin(ogen) staining}

Paraffin-embedded heart sections were deparaffinized with antigen retrieval. Slides were blocked with 3\% bovine serum albumin (Sigma Ardrich, St. Louis, MO, USA) in phosphate-buffered saline (Thermo Fisher Scientific, Waltham, MA, USA) for $30 \mathrm{~min}$ at room temperature. Sections were incubated overnight at $4{ }^{\circ} \mathrm{C}$ with $10 \mu \mathrm{g} \mathrm{mL}{ }^{-1}$ peroxidase labeled anti-fibrin antibody (clone 59D8), a gift from Dr. Charles T. Esmon, Oklahoma Medical Research Foundation. Fibrin staining (brown) was detected using the DAB substrate (Agilent Technology,), which was added for $2.5 \mathrm{~min}$. Sections were counterstaining with hematoxylin (Thermo Fisher Scientific) for $1 \mathrm{~min}$.

\subsection{Measurement of inflammatory mediators and myeloperoxidase}

Hearts were extracted immediately after euthanizing the mice and cut along the short axis at the level of the LAD ligation. The section below the LAD ligation was used for protein analysis. Hearts were homogenized in RIPA buffer with phosphatase and protease inhibitors as described $[13,37]$. IL-6, IL-1 $\beta$, and myeloperoxidase (MPO) protein levels in the hearts were measured with ELISA kits (R\&D systems, Minneapolis, MN).

\subsection{Measurement of plasma cardiac troponin-I}

Blood was collected from the retroorbital sinus into sodium citrate (final concentration, $0.38 \%$ ). The troponin-I concentration in plasma was measured using a mouse cardiac troponin-I ELISA kit (Life Diagnostics, West Chester, PA) [15,37].

\subsection{Measurement of infarct size}

For the measurement of infarct size at day 1, whole hearts were cut into $1 \mathrm{~mm}$ slices in the short axis. Viable and necrotic and apoptotic areas were identified by incubating the hearts in $1 \% 2,3,5-$ Triphenyltetrazolium chloride (TTC) (Sigma-Aldrich) for $30 \mathrm{~min}$ at $37^{\circ} \mathrm{C}$ 

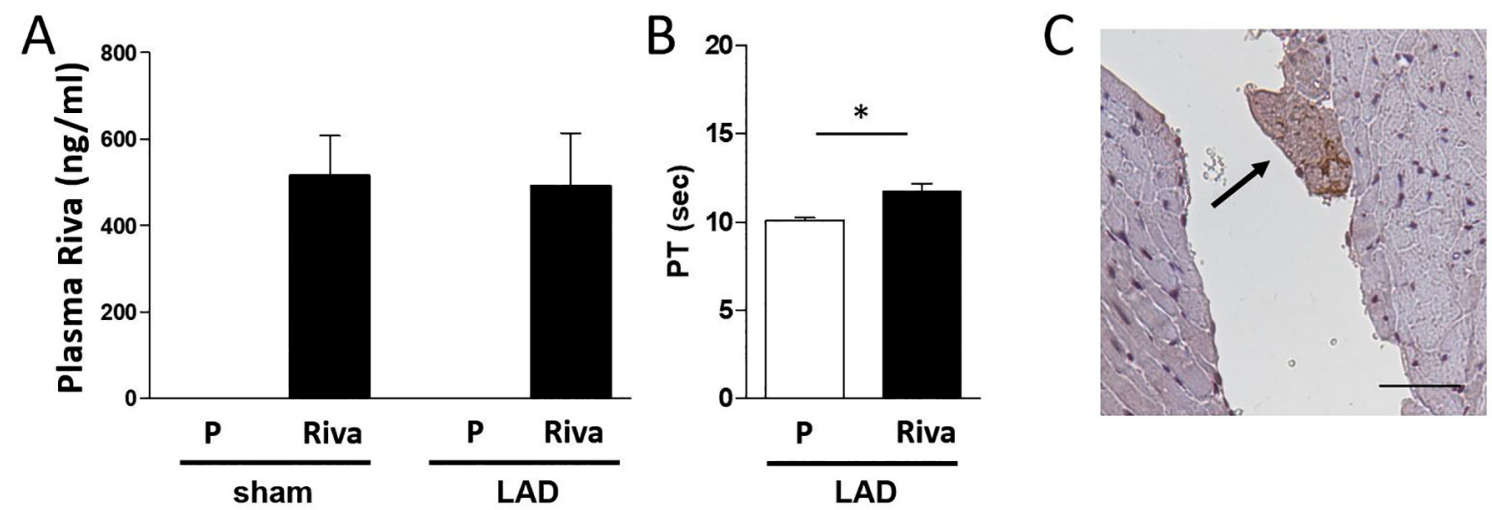

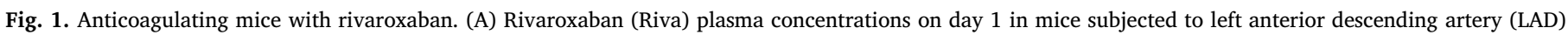

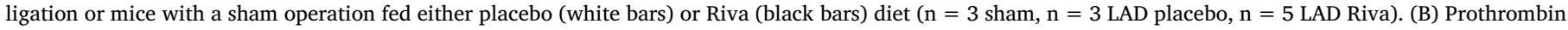

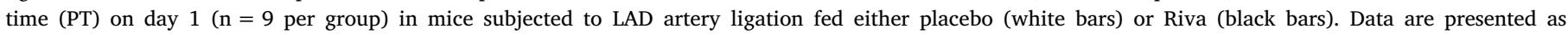

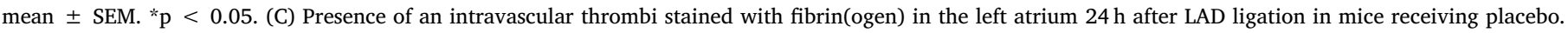
Original magnification $\times 200$, bar $=100 \mu \mathrm{m}$.

followed by $4 \%$ paraformaldehyde for $30 \mathrm{~min}$ at room temperature. Each section was weighed and photographed under a SZX12 microscope (Olympus Corporation, Tokyo, Japan) from both sides. The total area and necrotic area were traced and calculated by computer planimetry (Image $\mathrm{J}, \mathrm{NIH}$, version 1.21). Total infarct size was calculated as $\left[\left(\mathrm{A}_{1} \mathrm{xW}_{1}\right)+\left(\mathrm{A}_{2} \mathrm{xW}_{2}\right)+\ldots+\left(\mathrm{A}_{\mathrm{n}} \mathrm{xW}_{\mathrm{n}}\right)\right] /\left[\left(\mathrm{T}_{1} \mathrm{x}_{\mathrm{W}} 1\right)+\left(\mathrm{T}_{2} \mathrm{xW}_{2}\right)+\ldots+\right.$ $\left(\mathrm{T}_{\mathrm{n}} \mathrm{xW}\right)$, for $\mathrm{n}$ slices, where $\mathrm{A}$ is the area of infarct for the slice denoted by the subscript, $\mathrm{T}$ is the total area of the slice, and $\mathrm{W}$ is the weight of the respective slice.

For the measurement of infarct size at day 28, five sections were prepared from each heart and stained with Masson's trichrome. The section with the largest infarct diameter was chosen and the blue fibrotic area of the infarcted myocardium and the red area of the unaffected myocardium were analyzed by computer planimetry and the percentage of scar to the total area was calculated.

\subsection{Echocardiography}

Echocardiography was performed prior to cardiac injury and after cardiac injury on days 3, 7 and 28 using a Vevo 2100 ultrasonographic system (VisualSonics, Toronto, CA). Left ventricle (LV) function was measured by M-mode echocardiography derived from the long-axis parasternal plane at the mid-ventricular level. Interventricular septum thickness, posterior wall thickness and left ventricular diameter were measured in systole and diastole. The ejection fraction (EF) and percentage of fractional shortening (FS) were calculated from the measured dimensions. Echocardiography data were read and interpreted by W.B., who is a board-certified cardiologist.

\subsection{Data analysis}

All statistical analyses were performed using GraphPad Prism, version 5.0 (GraphPad Software Inc., La Jolla, CA) or SAS v9.2 (SAS, Cary, NC). Normally distributed data with equal variance was analyzed by one or two way ANOVA (multiple group comparison; Bonferroni Post Hoc analysis corrected for repeated measures over time) or a two-tailed Student's $t$-test (two group comparisons) where appropriate. Data are expressed as mean values \pm the standard error of the mean. Nominal data was analyzed by Chi-squared test. Log-rank analysis was used to determine statistical significance of the survival of the different mice. Differences were determined to be statistically significant at a $p$ value of $<0.05$.

\section{Results}

\subsection{Anticoagulant activity of mice receiving rivaroxaban}

Cardiac injury was induced in mice by permanent ligation of the LAD. The mice were given either placebo or rivaroxaban chow starting immediately after LAD ligation. Mice started eating chow 6-8 h after surgery. We observed similar plasma levels of rivaroxaban at day 1 in the sham and LAD groups (Fig. 1A). Similar data were observed at day 28 (data not shown). To assess the impact of rivaroxaban on coagulation, we measured the PT. The PT is considered more sensitive than the activated partial thromboplastin time to determine the anticoagulant effect of rivaroxaban [32]. Rivaroxaban significantly increased the PT 1 day after LAD ligation compared with the group receiving placebo (Fig. 1B). Next, we determined if rivaroxaban affected intravascular coagulation. We observed intravascular thrombi in 3 of 4 LAD ligated mice receiving placebo ( 2 in the left atrium and one in a small vessel) compared with 0 of $4 \mathrm{LAD}$ ligated mice receiving rivaroxaban $(\mathrm{p}=0.03)$ (Fig. 1C). Taken together, these data indicate that the mice were anticoagulated with rivaroxaban after LAD ligation.

\subsection{Measurement of infarct size after LAD ligation}

To assess the extent of myocardial injury in our model, we measured infarct size 1 day after LAD ligation. Importantly, there was no difference in infarct size between the mice receiving rivaroxaban or placebo (Fig. 2A). Plasma troponin-I was used as a surrogate marker of infarct size after LAD ligation. Again, there was no difference in troponin-I levels between rivaroxaban and placebo treated mice after LAD ligation (Fig. 2B). Consistent with previous reports [38,39], we observed a high rate of mortality (40\%) after surgery between days 4 and 6 due to rupture of the LV (Supplementary Fig. 1). However, there was no difference in mortality between the two groups.

We also measured the infarct size at day 28 . In contrast to the results from day 1 , we observed a significantly smaller infarct in the mice treated with rivaroxaban compared with mice that received placebo (Fig. 2C and D). This indicates that rivaroxaban is inhibiting the expansion of the initial infarct.

\subsection{Effect of rivaroxaban on inflammation and neutrophil recruitment after LAD ligation}

We measured several inflammatory cytokines in the hearts of mice 2 days after LAD ligation. There were no significant differences in the levels of IL-6, IL-1 $\beta$, and TNF- $\alpha$ in injured mice treated with 

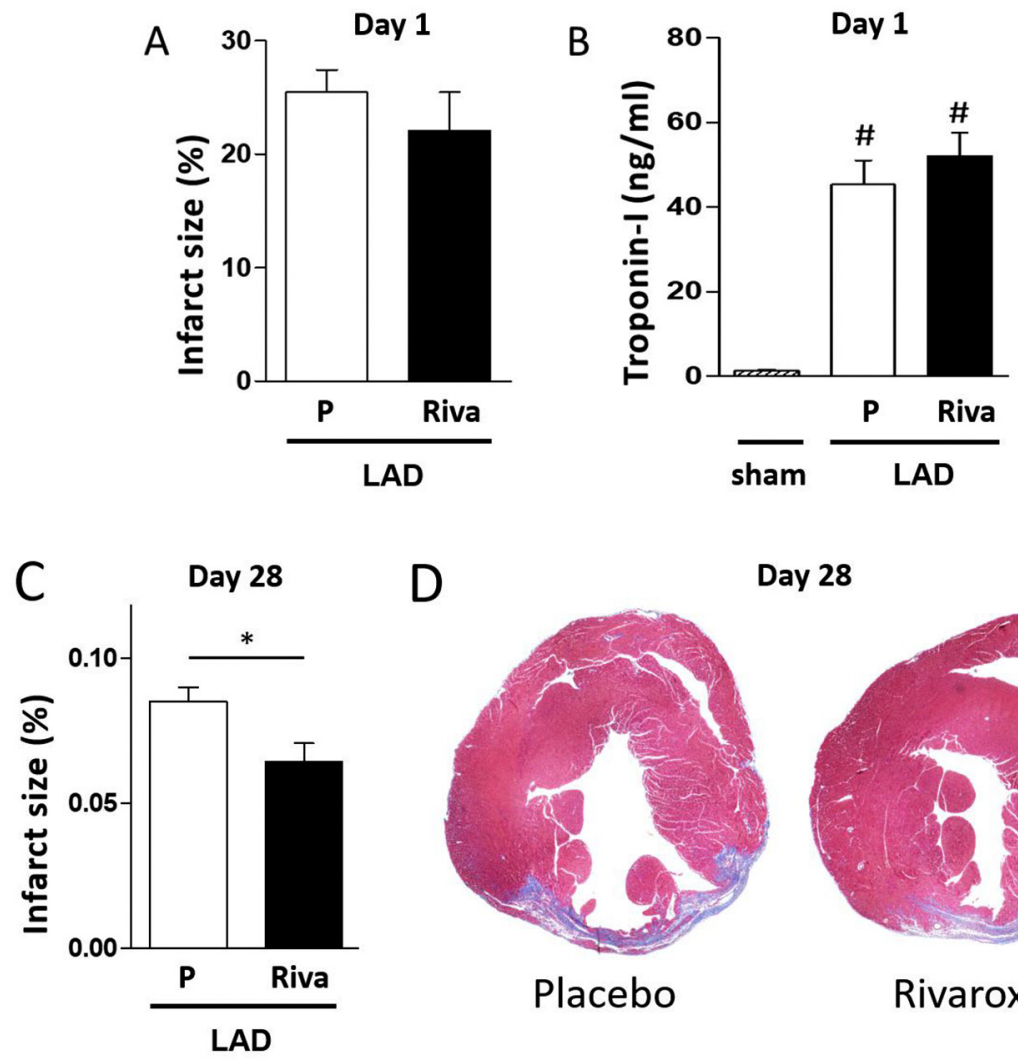

D

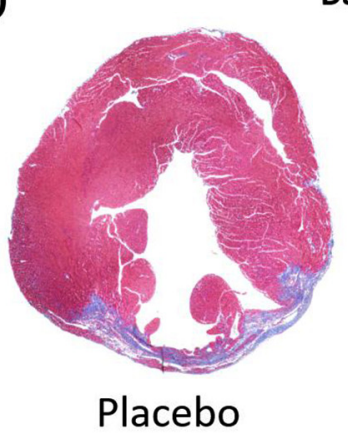

Day 28
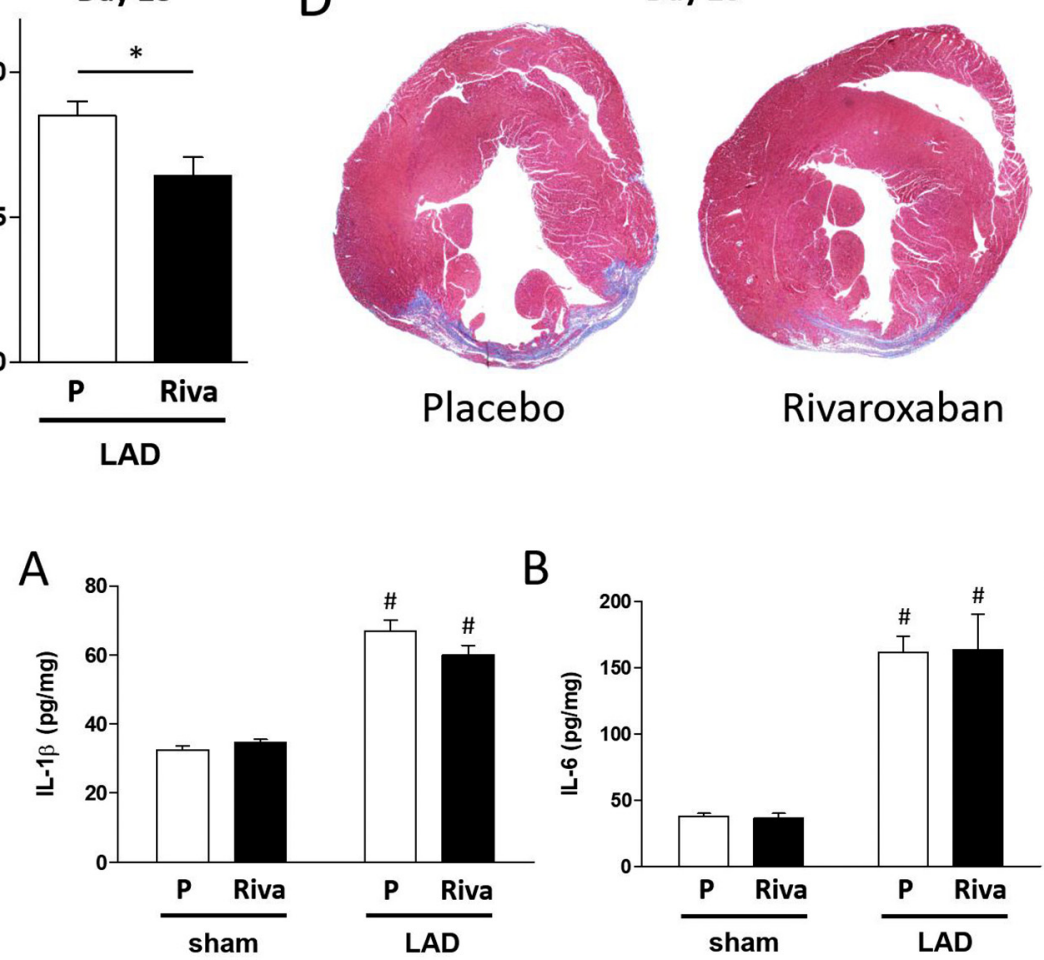

B

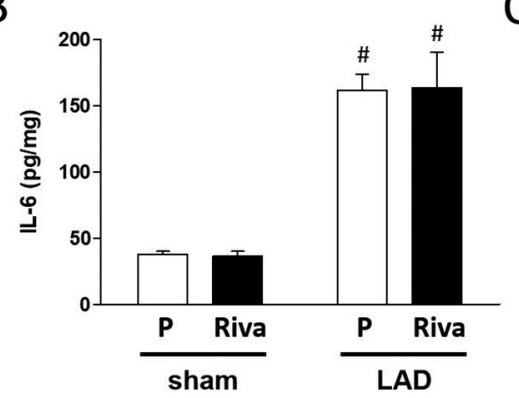

Fig. 2. Effect of rivaroxaban on infarct size at day 1 and 28. (A) Comparison of infarct size on day 1 in mice subjected to left anterior descending artery (LAD) ligation and treated with either placebo $(\mathrm{n}=7)$ or rivaroxaban $(n=6)$. Infarct size was measured with computer planimetry on $1 \mathrm{~mm}$ heart slices stained with 2,3,5Triphenyltetrazolium chloride. (B) Troponin-I levels increased due to LAD ligation. Both placebo $(n=18)$ and rivaroxaban treated mice $(n=14)$ had significantly higher troponin-I levels after LAD ligation compared to sham operated mice $(n=6)$ on day 1 . There was no difference between placebo and Riva treated mice with LAD ligation. Placebo: white bars, rivaroxaban: black bars. Data are presented as mean \pm SEM. \#p $<0.05$ vs. sham. (C) Infarct size at day 28 in mice treated with either placebo (white bar) $(n=12)$ or rivaroxaban (black bar) $(\mathrm{n}=15)$. Data is presented as mean \pm SEM. ${ }^{*} \mathrm{p}<0.05$. (D) Representative images of hearts from mice 28 days after LAD ligation and treated with either rivaroxaban or placebo.

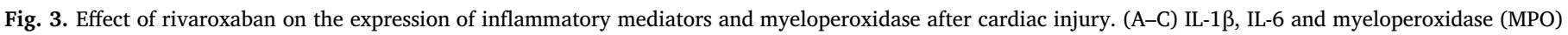

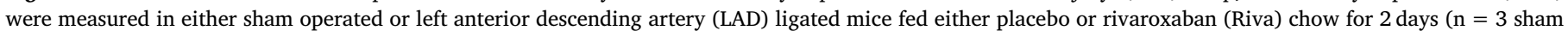

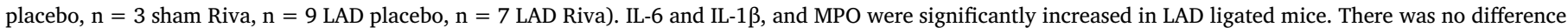

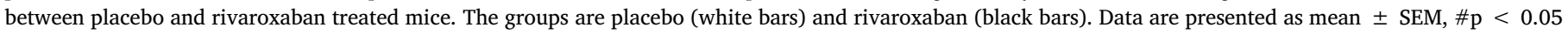
versus same treatment of sham mice.

rivaroxaban compared to those treated with placebo (Fig. 3A-C). We also measured MPO levels as a marker of neutrophil infiltration. Again, there was no difference in the levels of MPO between the two groups (Fig. 3D). These data suggest that rivaroxaban does not significantly reduce the expression of inflammatory mediators or the recruitment of neutrophils two days after LAD ligation.

\subsection{Effect of rivaroxaban on heart hypertrophy}

The heart weight/tibia lengths (HW:TL) ratio was calculated as a marker of hypertrophy and was found to be significantly increased at 7 and 28 days after LAD ligation in mice receiving placebo compared to sham operated mice (Fig. 4). Mice treated with rivaroxaban after LAD ligation had less hypertrophy than the placebo group but the difference was not statistically significant (Fig. 4).

\subsection{Rivaroxaban preserves cardiac function after LAD ligation}

To determine the effect of rivaroxaban on cardiac remodeling, we assessed cardiac function and wall thickness by echocardiography prior to LAD ligation and 3, 7 and 28 days after LAD ligation in mice given placebo or rivaroxaban on day 0 . The EF and FS of placebo-treated mice subjected to LAD ligation were significantly reduced compared to sham operated mice beginning on day 3 and continuing up to day 28 after LAD ligation (Fig. 5A) (Table 1). Mice treated with rivaroxaban had significantly less reduction in the EF and FS compared to mice receiving placebo (Fig. 5A) (Table 1). This effect was evident on day 3 and continued throughout the 28-day experimental period (Fig. 5A) (Table 1).

To assess the effects of the infarct on subsequent remodeling, the interventricular septum (IVS), the opposing left ventricular posterior wall (LVPW), and the left ventricular internal diameter (LVID) were measured by echocardiography. The infarcted IVS, which is directly 

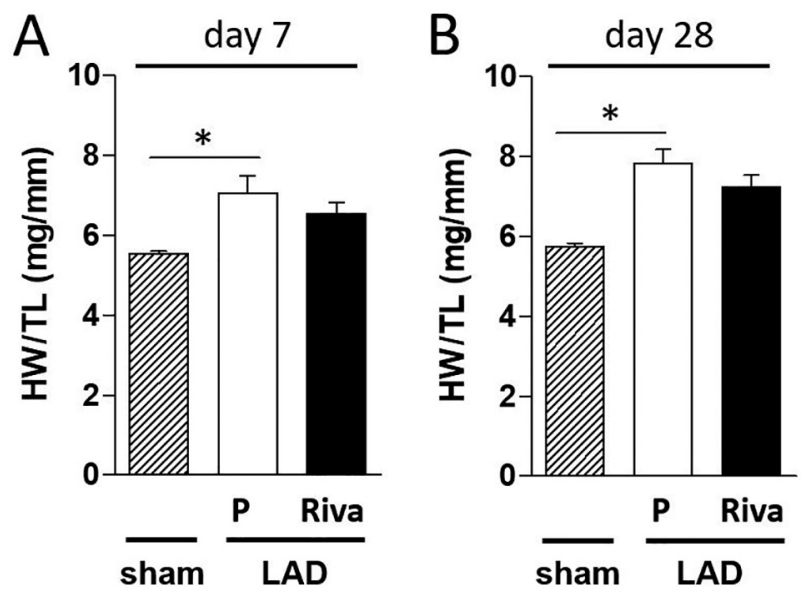

Fig. 4. Effects of rivaroxaban on hypertrophy after cardiac injury. Hearts and lungs of sham operated mice and mice subjected to left anterior descending artery (LAD) ligation and treated with placebo or rivaroxaban (Riva) were analyzed on day 7 ( $\mathrm{n}=3$ sham, $\mathrm{n}=9$ placebo, $\mathrm{n}=9$ Riva) or day $28(\mathrm{n}=3$ sham, $n=6$ placebo, $n=4$ Riva). The heart weight (HW) was measured and normalized to tibia length (TL). There was an increase in heart hypertrophy with LAD ligation at both time points but no statistical difference between the two treatments was observed. Sham operated (striped bars), placebo/LAD ligated (white bars) rivaroxaban/LAD (black bars). Data are presented as mean \pm SEM; ${ }^{*} \mathrm{p}<0.05$.

supplied by the LAD, was severely thinned in hearts of both placebo and rivaroxaban treated mice (Table 2). The LVPW was severely thinned in placebo treated mice indicating global thinning of the heart. However, the LVPW was thicker in rivaroxaban treated mice compared to controls, suggesting that rivaroxaban may reduce the impairment of heart function by protecting myocardium remote from the infarct (Table 2). In addition, rivaroxaban treated mice had significantly less heart dilation as indicated by a lower LVID compared to placebo treated mice (Table 2). The differences between treatment groups were observed as early as day 7 and persisted through day 28. These data suggest that treatment with rivaroxaban after LAD ligation preserves EF and FS by reducing pathologic remodeling of the LVPW and by reducing LV dilation.

To model the effect of rivaroxaban in patients with heart failure, we performed a second experiment in which rivaroxaban was administered beginning on day 3 after LAD ligation. Heart function was assessed by echocardiography prior to LAD ligation and 3, 7 and 28 days after LAD ligation. In contrast to the results observed when rivaroxaban was given to the mice on day 0 , there was no protective effect of rivaroxaban when it was given on day 3 (Fig. 5B). These data indicate that rivaroxaban needs to be administered within the first three days after LAD occlusion to exert its beneficial effects.
Table 1

Echocardiographic data at baseline and after cardiac injury.

\begin{tabular}{|c|c|c|c|c|c|}
\hline & & Sham & LAD/P & $\mathrm{LAD} / \mathrm{R}$ & $\begin{array}{l}\mathrm{LAD} / \mathrm{P} \text { vs LAD/ } \\
\mathrm{R}\end{array}$ \\
\hline \multirow[t]{4}{*}{$\mathrm{EF}(\%)$} & d 0 & $83.03 \pm 2.7$ & $82.9 \pm 0.56$ & $83.53 \pm 1.91$ & \\
\hline & d 3 & $83.63 \pm 2.74$ & $36.9 \pm 2.78$ & $51.25 \pm 7.51$ & * \\
\hline & d 7 & $78.94 \pm 3.76$ & $29.61 \pm 4.38$ & $50.98 \pm 6.37$ & $* *$ \\
\hline & d 28 & $87.7 \pm 0.81$ & $19.61 \pm 3.86$ & $40.86 \pm 3.06$ & $* * * *$ \\
\hline \multirow[t]{4}{*}{ FS (\%) } & d 0 & $49.89 \pm 3.1$ & $49.58 \pm 0.82$ & $50.71 \pm 2.12$ & \\
\hline & d 3 & $50.78 \pm 2.95$ & $17.56 \pm 1.52$ & $26.32 \pm 4.94$ & * \\
\hline & d 7 & $45.97 \pm 3.57$ & $14.05 \pm 2.25$ & $25.79 \pm 3.86$ & $* *$ \\
\hline & d 28 & $55.91 \pm 1.15$ & $9.13 \pm 1.88$ & $20.08 \pm 1.67$ & $* * * *$ \\
\hline
\end{tabular}

Mice underwent either a sham operation $(n=3)$ or left anterior descending $(\mathrm{LAD})$ artery ligation and were treated with placebo $(\mathrm{P})(\mathrm{n}=9)$ or rivaroxaban $(\mathrm{R})(\mathrm{n}=9)$ starting on day 0 . Echocardiography was performed prior to cardiac injury on day 0 and after cardiac injury on day 3,14, and 28. Ejection fraction (EF) and fractional shortening (FS) were calculated based on M-mode measurements. Data are presented as mean \pm SEM. 2-way ANOVA; *p $<0.05$, ${ }^{* * \mathrm{p}}<0.01,{ }^{* * * \mathrm{p}}<0.001$.

\subsection{Effect of rivaroxaban on cardiac function after $L A D$ ligation in PAR-2 deficient mice}

Since FXa can activate PAR-2, we determined the effect of rivaroxaban on cardiac remodeling in PAR-2 ${ }^{-/-}$mice subjected to LAD ligation. As expected from our previous study [15], placebo-treated PAR$2^{-/-}$mice subjected to LAD ligation had less impairment of LV function compared with placebo-treated PAR-2 $2^{+/+}$mice (Fig. 6). Rivaroxaban reduced the impairment of cardiac function in PAR $-2^{+/+}$mice but not in PAR-2 $2^{-/-}$mice after LAD ligation (Fig. 6).

\section{Discussion}

In this study, we found that early but not late administration of the FXa inhibitor rivaroxaban reduces the impairment of cardiac function in a mouse model of LAD ligation. Rivaroxaban is an anticoagulant that specifically targets FXa. However, rivaroxaban may also reduce inflammation by inhibiting FXa activation of PAR-2. We found that rivaroxaban prolonged the PT and reduced intravascular coagulation, including formation of thrombi in the left atrium, in mice subjected to LAD ligation indicating the mice were anticoagulated. Other studies have shown that rivaroxaban inhibits coagulation in mouse models. For instance, it reduced TF-induced thrombosis in mice subjected to cardiac I/R injury and also reduced thrombosis in a catheter model [23,24]. Another study showed that rivaroxaban abolished left atrial thrombus formation in a mouse model of transverse aortic constriction that induces pressure-overload-induced cardiac hypertrophy [27]. Therefore, the protective effect of rivaroxaban may be, in part, due to its anticoagulant activity.
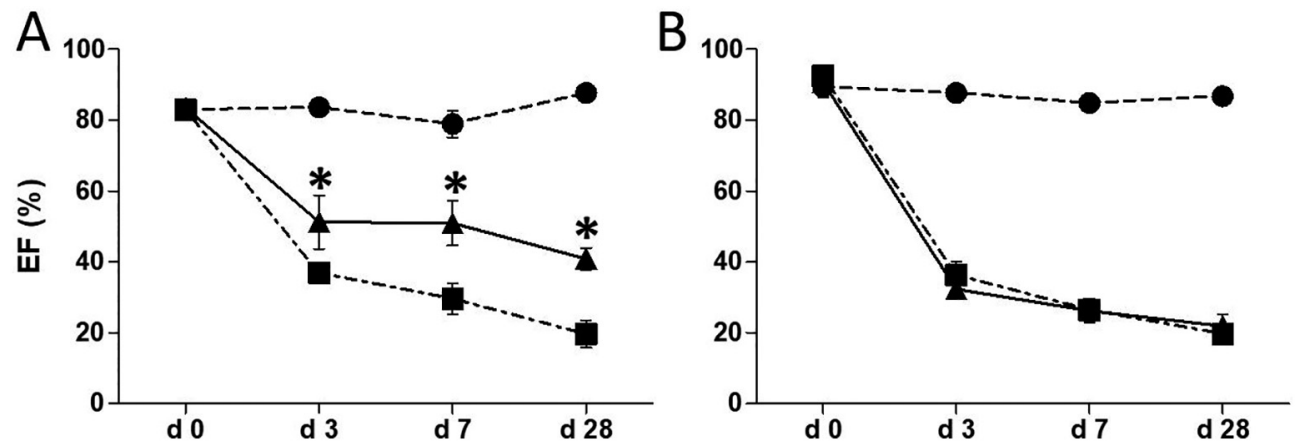

Fig. 5. Rivaroxaban preserves ejection fraction by decreasing cardiac remodeling. Echocardiography was performed on the different groups of mice on days $0,3,7$ and 28. (A) Mice underwent either a sham operation $(n=3)$ or left anterior descending artery (LAD) ligation and were treated with placebo $(n=9)$ or rivaroxaban $(n=9)$. Placebo mice had a significantly reduced ejection fraction (EF) compared to sham operated mice at all time points. Mice that underwent LAD ligation but were fed Rivaroxaban (Riva) chow had a significantly preserved EF compared to placebo mice on days 3, 7 and 28. (B) Mice underwent sham operation $(n=3)$ or underwent LAD ligation with placebo $(n=11)$ or rivaroxaban treatment $(n=9)$ beginning on day 3 after surgery. No difference between placebo and rivaroxaban treated groups was observed. Sham: circles; placebo/LAD: squares; rivaroxaban/ LAD: triangles. Data are presented as mean \pm SEM; *p $<0.05$. 
Table 2

Echocardiographic measurements at baseline and after cardiac injury.

\begin{tabular}{llllll}
\hline \multicolumn{3}{c}{ Sham } & LAD/P & LAD/R & $\begin{array}{l}\text { LAD/P vs } \\
\text { LAD/R }\end{array}$ \\
\hline \multirow{2}{*}{ IVS mm } & d 0 & $1.71 \pm 0.04$ & $1.75 \pm 0.12$ & $1.57 \pm 0.06$ & \\
& d 3 & $1.59 \pm 0.07$ & $0.78 \pm 0.1$ & $1.12 \pm 0.29$ & \\
& d 7 & $1.75 \pm 0.09$ & $0.54 \pm 0.03$ & $0.9 \pm 0.26$ & \\
& d 28 & $1.73 \pm 0.05$ & $0.34 \pm 0.06$ & $0.54 \pm 0.19$ & \\
LVID (mm) & d 0 & $1.18 \pm 0.11$ & $1.23 \pm 0.08$ & $1.29 \pm 0.06$ & \\
& d 3 & $1.25 \pm 0.13$ & $3.27 \pm 0.2$ & $2.63 \pm 0.4$ & \\
& d 7 & $1.4 \pm 0.23$ & $4.41 \pm 0.47$ & $2.78 \pm 0.37$ & $*$ \\
LVPW (mm) & d 28 & $1.24 \pm 0.07$ & $5.11 \pm 0.39$ & $3.77 \pm 0.21$ & $* *$ \\
& d 0 & $1.72 \pm 0.05$ & $1.64 \pm 0.06$ & $1.58 \pm 0.06$ & \\
& d 3 & $1.52 \pm 0.04$ & $1.16 \pm 0.12$ & $1.38 \pm 0.2$ & \\
& d 7 & $1.47 \pm 0.04$ & $0.56 \pm 0.13$ & $1.23 \pm 0.14$ & $*$ \\
& d 28 & $1.73 \pm 0.05$ & $0.61 \pm 0.13$ & $1.27 \pm 0.17$ & $* *$
\end{tabular}

Mice underwent either a sham operation $(n=3)$ or left anterior descending $(\mathrm{LAD})$ artery ligation and were treated with placebo $(\mathrm{n}=9)$ or rivaroxaban $(\mathrm{n}=9)$ starting on day 0 . Echocardiography was performed prior to cardiac injury on day 0 and after cardiac injury on day 3,14, and 28. Measurements were performed in M-mode in systole. IVS: Interventricular septum; LVID: Left ventricular internal diameter; LVPW: Left ventricular posterior wall. Data are presented as mean \pm SEM. 2-way ANOVA; $* \mathrm{p}<0.05$, ${ }^{* *} \mathrm{p}<0.01$, $* * * \mathrm{p}<0.001$

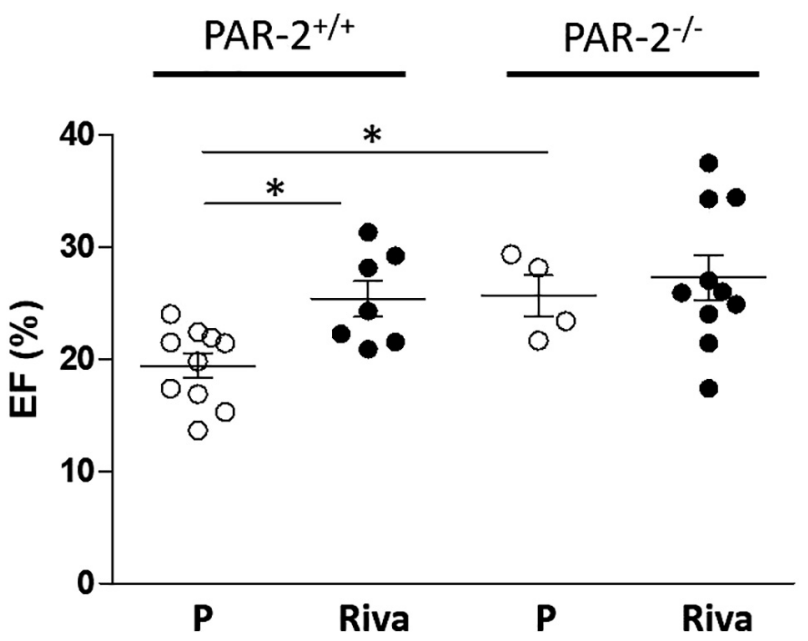

Fig. 6. Effect of rivaroxaban on cardiac function in PAR-2 $2^{-/}$mice after LAD ligation. PAR-2 wild-type (WT) and PAR-2 ${ }^{-/-}$mice were subjected to left anterior descending (LAD) artery ligation and were fed either placebo (C) or rivaroxaban (Riva) chow beginning on day 0 . Echocardiography was performed on all mice on day 28. Placebo treated PAR- $2^{-1-}$ mice $(n=4)$ had less reduction in EF compared to placebo treated PAR-2 WT mice $(n=10)$. Rivaroxaban treated PAR-2 WT mice $(\mathrm{n}=7)$ were similarly protected compared to placebo treated PAR- $2^{-/-}$mice. Rivaroxaban treated PAR- $2^{-/-}$mice ( $\mathrm{n}=10$ ) were not significantly protected in compared to placebo treated PAR$2^{-/-}$mice. The groups are PAR-2 WT (white bars) and PAR-2 ${ }^{-/-}$mice (black bars). Data are presented as mean \pm SEM; *p $<0.05$.

Rivaroxaban has been shown to be protective in a variety of mouse models, including transverse aortic constriction, atherosclerosis, and sickle cell disease [27,28,32,40]. Interestingly, rivaroxaban reduced inflammation in these models. In addition, edoxaban, another member of the class of oral FXa inhibitors, reduced inflammation and diabetic retinopathy in a mouse model [30]. Surprisingly, we found that rivaroxaban did not affect the expression of inflammatory mediators or MPO two days after LAD ligation. Two studies investigated the effect of the FXa inhibitor fondaparinux in a rat model of cardiac I/R injury $[8,9]$. Interestingly, fondaparinux reduced infarct size without affecting inflammation. The protective effect of fondaparinux was dependent on STAT-3 phosphorylation and was associated with increase expression of thrombomodulin and endothelial cell protein $C$ receptor $[8,9]$.
In addition to its central role in blood coagulation, FXa can also activate PAR-2 [12]. We and others have shown that PAR-2 activation induces hypertrophy of cultured cardiomyocytes [15,41]. We found that PAR-2 $2^{-1-}$ mice had reduced inflammation in the cardiac I/R model compared to controls [13]. Furthermore, we showed that PAR$2^{-/-}$mice have less cardiac impairment in the LAD model [15]. In other studies, PAR-2 ${ }^{-/-}$mice had reduced diabetic nephropathy, atherosclerosis and inflammation in the sickle cell disease model $[30,32,42]$. The fact that the phenotype of PAR-2 ${ }^{-/-}$mice is similar to wild-type mice treated with rivaroxaban in these three models suggests that rivaroxaban may be acting by reducing pathological PAR-2 signaling.

Consistent with these studies, we found that rivaroxaban did not provide additional protection to PAR- $2^{-/-}$mice in the LAD model. This suggests that rivaroxaban may reduce expansion of the infarct after LAD ligation, in part, by reducing pathological PAR-2 signaling. However, we cannot exclude the possibility that there are two parallel pathways, namely a FXa-thrombin-fibrin pathway and a PAR-2 pathway, that independently contribute to cardiac injury via different mechanisms. Indeed, PAR-2 is activated by a variety of proteases, such as tryptase, that are present during cardiac injury [43].

Taken together, our data suggest that rivaroxaban may be cardioprotective via a combination of two activities - its anticoagulant activity and its ability to inhibit pathological FXa-PAR-2 signaling. Inhibition of coagulation would reduce intravascular thrombi and help preserve blood flow to cardiomyocytes in the border zone around the infarct. Reducing FXa-PAR-2 signaling would reduce hypertrophy of cardiomyocytes and cardiac remodeling.

Supplementary data to this article can be found online at https:// doi.org/10.1016/j.thromres.2018.05.015.

\section{Competing interests statement}

None.

\section{Acknowledgments}

This research was supported, in part, by a grant from Janssen Research and Development, LLC. We would like to thank Ying Zhang, Clare Schmedes and Madison Woo for technical support. The surgery and echocardiography were performed in the Animal Surgery Core Lab of the McAllister Heart Institute at UNC.

\section{References}

[1] M.G. Sutton, N. Sharpe, Left ventricular remodeling after myocardial infarction: pathophysiology and therapy, Circulation 101 (2000) 2981-2988.

[2] W.Y. Lew, Z.Y. Chen, B. Guth, J.W. Covell, Mechanisms of augmented segment shortening in nonischemic areas during acute ischemia of the canine left ventricle, Circ. Res. 56 (1985) 351-358.

[3] M. Jessup, S. Brozena, Heart failure, N. Engl. J. Med. 348 (2003) 2007-2018.

[4] J.H. Erlich, E.M. Boyle, J. Labriola, J.C. Kovacich, R.A. Santucci, C. Fearns, E.N. Morgan, W. Yun, T. Luther, O. Kojikawa, T.R. Martin, T.H. Pohlman, E.D. Verrier, N. Mackman, Inhibition of the tissue factor-thrombin pathway limits infarct size after myocardial ischemia-reperfusion injury by reducing inflammation, Am. J. Pathol. Am. Soc. Invest. Pathol. 157 (2000) 1849-1862.

[5] P. Golino, M. Ragni, P. Cirillo, A. Scognamiglio, A. Ravera, C. Buono, A. Guarino, O. Piro, C. Lambiase, F. Botticella, M. Ezban, M. Condorelli, M. Chiariello, Recombinant human, active site-blocked factor VIIa reduces infarct size and noreflow phenomenon in rabbits, Am. J. Physiol. Heart Circ. Physiol. 278 (2000) H1507-16.

[6] R. Pawlinski, M. Tencati, C.R. Hampton, T. Shishido, T.A. Bullard, L.M. Casey, P. Andrade-Gordon, M. Kotzsch, D. Spring, T. Luther, J. Abe, T.H. Pohlman, E.D. Verrier, B.C. Blaxall, N. Mackman, Protease-activated receptor-1 contributes to cardiac remodeling and hypertrophy, Circulation 116 (2007) 2298-2306.

[7] S.T. Loubele, C.A. Spek, P. Leenders, Oerle R. van, H.L. Aberson, Voort D. van der, K. Hamulyak, L.C. Petersen, H.M. Spronk, Cate H. ten, Active site inhibited factor VIIa attenuates myocardial ischemia/reperfusion injury in mice, J. Thromb. Haemost. 7 (2009) 290-298.

[8] L. Macchi, Moussa W. Ben, S. Guillou, S. Tamareille, D. Lamon, D. Prunier, F. Prunier, The synthetic pentasaccharide fondaparinux attenuates myocardial ischemia-reperfusion injury in rats via STAT-3, Shock 41 (2014) 166-171. 
[9] S. Guillou, S. Tamareille, S. Giraud, G. Poitevin, D. Prunier-Mirebeau, P. Nguyen, F. Prunier, L. Macchi, Fondaparinux upregulates thrombomodulin and the endothelial protein $\mathrm{C}$ receptor during early-stage reperfusion in a rat model of myocardial infarction, Thromb. Res. 141 (2016) 98-103.

[10] M.J. Flick, X. Du, J.L. Degen, Fibrin(ogen)-alpha M beta 2 interactions regulate leukocyte function and innate immunity in vivo, Exp. Biol. Med. (Maywood) 229 (2004) 1105-1110.

[11] P. Petzelbauer, P.A. Zacharowski, Y. Miyazaki, P. Friedl, G. Wickenhauser, F.J. Castellino, M. Gröger, K. Wolff, K. Zacharowski, The fibrin-derived peptide Bbeta15-42 protects the myocardium against ischemia-reperfusion injury, Nat. Med. 11 (2005) 298-304.

[12] S.R. Coughlin, Protease-activated receptors in hemostasis, thrombosis and vascular biology, J. Thromb. Haemost. 3 (2005) 1800-1814.

[13] S. Antoniak, M. Rojas, D. Spring, Bullard T a, E.D. Verrier, B.C. Blaxall, N. Mackman, R. Pawlinski, Protease-activated receptor 2 deficiency reduces cardiac ischemia/reperfusion injury, Arterioscler. Thromb. Vasc. Biol. 30 (2010) 2136-2142.

[14] M.A. Kolpakov, K. Rafiq, X. Guo, B. Hooshdaran, T. Wang, L. Vlasenko, Y.V. Bashkirova, X. Zhang, X. Chen, S. Iftikhar, J.R. Libonati, S.P. Kunapuli, A. Sabri, Protease-activated receptor 4 deficiency offers cardioprotection after acute ischemia reperfusion injury, J. Mol. Cell. Cardiol. 90 (2016) 21-29.

[15] S. Antoniak, E.M. Sparkenbaugh, M. Tencati, M. Rojas, N. Mackman, R. Pawlinski, Protease activated receptor-2 contributes to heart failure, PLoS One 8 (2013) e81733.

[16] S.E. Warren, H.D. Royal, J.E. Markis, W. Grossman, R.G. McKay, Time course of left ventricular dilation after myocardial infarction: influence of infarct-related artery and success of coronary thrombolysis, J. Am. Coll. Cardiol. 11 (1988) 12-19.

[17] J.P. Cleutjens, J.C. Kandala, E. Guarda, R.V. Guntaka, K.T. Weber, Regulation of collagen degradation in the rat myocardium after infarction, J. Mol. Cell. Cardiol. 27 (1995) 1281-1292.

[18] E. Braunwald, Biomarkers in heart failure, N. Engl. J. Med. 358 (2008) 2148-2159.

[19] P.C. Westman, M.J. Lipinski, D. Luger, R. Waksman, R.O. Bonow, E. Wu, S.E. Epstein, Inflammation as a driver of adverse left ventricular remodeling after acute myocardial infarction, J. Am. Coll. Cardiol. 67 (2016) 2050-2060.

[20] S.D. Anker, S. von Haehling, Inflammatory mediators in chronic heart failure: an overview, Heart 90 (2004) 464-470.

[21] E. Perzborn, J. Strassburger, A. Wilmen, J. Pohlmann, S. Roehrig, K.-H. Schlemmer, A. Straub, In vitro and in vivo studies of the novel antithrombotic agent BAY 597939-an oral, direct Factor Xa inhibitor, J. Thromb. Haemost. 3 (2005) 514-521.

[22] J.I. Weitz, I.H. Jaffer, J.C. Fredenburgh, Recent Advances in the Treatment of Venous Thromboembolism in the Era of the Direct Oral Anticoagulants, F1000Research, 6 (2017), p. 985.

[23] M. Goto, S.-I. Miura, Y. Suematsu, Y. Idemoto, K. Takata, S. Imaizumi, Y. Uehara, K. Saku, Rivaroxaban, a factor Xa inhibitor, induces the secondary prevention of cardiovascular events after myocardial ischemia reperfusion injury in mice, Int. J. Cardiol. 220 (2016) 602-607.

[24] C.M. Terry, Y. He, A.K. Cheung, Rivaroxaban improves patency and decreases inflammation in a mouse model of catheter thrombosis, Thromb. Res. 144 (2016) 106-112.

[25] R. Morihara, T. Yamashita, S. Kono, J. Shang, Y. Nakano, K. Sato, N. Hishikawa, Y. Ohta, S. Heitmeier, E. Perzborn, K. Abe, Reduction of intracerebral hemorrhage by rivaroxaban after tPA thrombolysis is associated with downregulation of PAR-1 and PAR-2, J. Neurosci. Res. 95 (2017) 1818-1828.

[26] R. Ploen, L. Sun, W. Zhou, S. Heitmeier, M. Zorn, E. Jenetzky, R. Veltkamp, Rivaroxaban does not increase hemorrhage after thrombolysis in experimental ischemic stroke, J. Cereb. Blood Flow Metab. 34 (2014) 495-501.

[27] H. Kondo, I. Abe, A. Fukui, S. Saito, M. Miyoshi, K. Aoki, T. Shinohara, Y. Teshima, K. Yufu, N. Takahashi, Possible role of rivaroxaban in attenuating pressure-overload-induced atrial fibrosis and fibrillation, J. Cardiol. 71 (2017) 310-319.

[28] Q. Zhou, F. Bea, M. Preusch, H. Wang, B. Isermann, K. Shahzad, H.A. Katus, E. Blessing, Evaluation of plaque stability of advanced atherosclerotic lesions in apo E-deficient mice after treatment with the oral factor Xa inhibitor rivaroxaban,
Mediat. Inflamm. 2011 (2011) 432080

[29] N.-M. Wagner, T. Dressel, K. Schäfer, S. Konstantinides, Effect of the factor Xa inhibitor rivaroxaban on arterial thrombosis in wild-type and apolipoprotein E-deficient mice, Thromb. Res. 130 (2012) 793-798.

[30] Y. Oe, S. Hayashi, T. Fushima, E. Sato, K. Kisu, H. Sato, S. Ito, N. Takahashi, Coagulation factor Xa and protease-activated receptor 2 as novel therapeutic targets for diabetic nephropathy, Arterioscler. Thromb. Vasc. Biol. 36 (2016) 1525-1533.

[31] T.-C. Wu, J.-S. Chan, C.-Y. Lee, H.-B. Leu, P.-H. Huang, J.-S. Chen, S.-J. Lin, J.W. Chen, Rivaroxaban, a factor Xa inhibitor, improves neovascularization in the ischemic hindlimb of streptozotocin-induced diabetic mice, Cardiovasc. Diabetol. 14 (2015) 81.

[32] E.M. Sparkenbaugh, P. Chantrathammachart, J. Mickelson, Ryn J. van, R.P. Hebbel, D.M. Monroe, N. Mackman, N.S. Key, R. Pawlinski, Differential contribution of FXa and thrombin to vascular inflammation in a mouse model of sickle cell disease, Blood 123 (2014) 1747-1756.

[33] B.P. Damiano, W.M. Cheung, R.J. Santulli, W.P. Fung-Leung, K. Ngo, R.D. Ye, L. Darrow a, C.K. Derian, L. de Garavilla, Andrade-Gordon P. Cardiovascular responses mediated by protease-activated receptor-2 (PAR-2) and thrombin receptor (PAR-1) are distinguished in mice deficient in PAR-2 or PAR-1, J. Pharmacol. Exp. Ther. 288 (1999) 671-678.

[34] M.V.V. Kolk, D. Meyberg, T. Deuse, K.R. Tang-Quan, R.C. Robbins, H. Reichenspurner, S. Schrepfer, LAD-ligation: a murine model of myocardial in farction, J. Vis. Exp. 32 (2009) e1438.

[35] M.M. Samama, G. Contant, T.E. Spiro, E. Perzborn, C. Guinet, Y. Gourmelin, Flem L. Le, G. Rohde, J.L. Martinoli, Rivaroxaban Anti-Factor Xa Chromogenic Assay Field Trial Laboratories. Evaluation of the anti-factor Xa chromogenic assay for the measurement of rivaroxaban plasma concentrations using calibrators and controls, Thromb. Haemost. 107 (2012) 379-387.

[36] K. Tatsumi, S. Antoniak, S. Subramaniam, B. Gondouin, S.D. Neidich, M.A. Beck, J. Mickelson, D.M. Monroe, J.A. Bastarache, N. Mackman, Anticoagulation increases alveolar hemorrhage in mice infected with influenza A, Phys. Rep. 4 (2016).

[37] S. Antoniak, A.P. Owens, M. Baunacke, J.C. Williams, R.D. Lee, A. Weithäuser, P.A. Sheridan, R. Malz, J.P. Luyendyk, D.A. Esserman, J. Trejo, D. Kirchhofer, B.C. Blaxall, R. Pawlinski, M.A. Beck, U. Rauch, N. Mackman, PAR-1 contributes to the innate immune response during viral infection, J. Clin. Invest. 123 (2013) 1310-1322.

[38] B. Unsold, A. Kaul, M. Sbroggio, C. Schubert, V. Regitz-Zagrosek, M. Brancaccio, F. Damilano, E. Hirsch, M. van Bilsen, C. Munts, K. Sipido, V. Bito, E. Detre, N.M. Wagner, K. Schafer, T. Seidler, J. Vogt, S. Neef, A. Bleckmann, L.S. Maier, J.L. Balligand, C. Bouzin, R. Ventura-Clapier, A. Garnier, T. Eschenhagen, A. ElArmouche, R. Knoll, G. Tarone, G. Hasenfuss, Melusin protects from cardiac rupture and improves functional remodelling after myocardial infarction, Cardiovasc. Res. 101 (2014) 97-107.

[39] A. Ducharme, S. Frantz, M. Aikawa, E. Rabkin, M. Lindsey, L.E. Rohde, F.J. Schoen, R.A. Kelly, Z. Werb, P. Libby, R.T. Lee, Targeted deletion of matrix metalloproteinase-9 attenuates left ventricular enlargement and collagen accumulation after experimental myocardial infarction, J. Clin. Invest. 106 (2000) 55-62.

[40] T. Hara, D. Fukuda, K. Tanaka, Y. Higashikuni, Y. Hirata, S. Nishimoto, S. Yagi, H. Yamada, T. Soeki, T. Wakatsuki, M. Shimabukuro, M. Sata, Rivaroxaban, a nove oral anticoagulant, attenuates atherosclerotic plaque progression and destabilization in ApoE-deficient mice, Atherosclerosis 242 (2015) 639-646.

[41] A. Sabri, G. Muske, H. Zhang, E. Pak, A. Darrow, P. Andrade-Gordon, S.F. Steinberg, Signaling properties and functions of two distinct cardiomyocyte protease-activated receptors, Circ. Res. 86 (2000) 1054-1061.

[42] S.M. Jones, A. Mann, K. Conrad, K. Saum, D.E. Hall, L.M. McKinney, N. Robbins, J. Thompson, A.D. Peairs, E. Camerer, K.J. Rayner, M. Tranter, N. Mackman, A.P. Owens, PAR2 (protease-activated receptor 2) deficiency attenuates atherosclerosis in mice, Arterioscler. Thromb. Vasc. Biol. (2018) (in press).

[43] C.D. Major, R.J. Santulli, C.K. Derian, P. Andrade-Gordon, Extracellular mediators in atherosclerosis and thrombosis: lessons from thrombin receptor knockout mice, Arterioscler. Thromb. Vasc. Biol. 23 (2003) 931-939. 